\title{
IMAGEN E IDENTIDAD CORPORATIVA: EJEMPLOS DE CONSTRUCCIÓN DE LA IDENTIDAD VISUAL
}

\author{
Lic. Tomás Carlos Barriga Barriga \\ Profesor del Departamento de Ciencias Humanas de la \\ Universidad Nacional Agraria La Molina \\ tbarriga@lamolina.edu.pe
}

\begin{abstract}
Resumen
Este artículo analiza los conceptos de identidad e imagen en la empresa para proponer mecanismos de construcción de una imagen visual. Propone ejemplos y aporta ideas para el mejor manejo de estos dos conceptos en la comunicación empresarial.
\end{abstract}

Palabras claves: Imagen, logotipo, identidad visual, identidad corporativa, identidad visual, cultura organizacional.

\begin{abstract}
This article analyzes the concepts of identity and image in the company to propose mechanisms of construction of a visual image. It proposes examples and it contributes ideas for the best handling in these two concepts in the managerial communication.
\end{abstract}

Key words: Image, logo, visual identity, corporate identity, visual identity, organizational culture. 


\section{Introducción}

La comunicación corporativa estudia el flujo y los canales de comunicación de una organización tanto interna como externamente. En su estudio se enfrenta a los dos pilares de la comunicación y una corporación: la identidad corporativa y la imagen corporativa.

Una empresa, al igual que una persona, tiene un conjunto de cualidades que la hacen ser quien es (identidad): su aspecto, sus valores, sus costumbres, sus ventajes y defectos diferenciales. Al igual que una persona, también proyecta un conjunto de percepciones hacia los demás (imagen) que puede ser congruente o no con su identidad.

Toda organización tiene identidad e imagen, pero en la mayoría de los casos ambas son espontáneas, fruto de las tradiciones y costumbres aleatorias, de las influencias de quienes la dirigen y, en algunos casos, de la desorganización.

Una estrategia de comunicación organizacional debe establecer, en primer lugar, cuáles son los elementos de identidad adecuados para el funcionamiento de la empresa. Si estos no se corresponden con la realidad, será necesario empezar por modificar lo que la empresa es. Solo entonces se podrá proyectar una imagen deseada y sustentada en la realidad.

Las empresas que poseen una mala identidad y se esfuerzan por proyectar una buena imagen solo logran, a la larga, decepcionar y frustrar. Las que poseen una buena identidad pero no proyectan una imagen adecuada, no son consideradas por el público (digamos que nadie sabe lo buenas que son).

La imagen debe ser una proyección de la identidad. El objetivo final de una estrategia corporativa: ser el mejor y hacer que todos lo sepan.

\section{Identidad corporativa}

Cuando se empezaron a definir y estructurar las estrategias de comunicación corporativa se empezó a utilizar el término identidad corporativa como sinónimo de logotipo, estilo corporativo y otras formas de simbolismo utilizadas por una organización. Hoy

el concepto se ha extendido, y se ha hecho más amplio, ahora se refiere a la forma en la que una empresa se presenta mediante el uso de simbolos, comunicación y comportamientos. Estos tres elementos constituyen el llamado mix de identidad corporativa (IC). Todos los elementos del mix de IC pueden usarse para presentar la personalidad de una empresa tanto en forma interna como externa, según la filosofía de la empresa (1)

Para su mejor estudio y debido a la diversidad de autores que clasifican la identidad corporativa de muchas maneras, vamos a dividirla desde el punto de vista de la comunicación visual en identidad visual e identidad no visual.

\subsection{Identidad visual}

Es un sistema de signos visuales que tiene por objeto facilitar el reconocimiento y la 
recordación de una empresa. Este sistema está compuesto por dos niveles: el lingüístico y el icónico.

1.1.1 Lingüístico: Es el nombre de la empresa, o marca gráfica, incluyendo la tipografía.

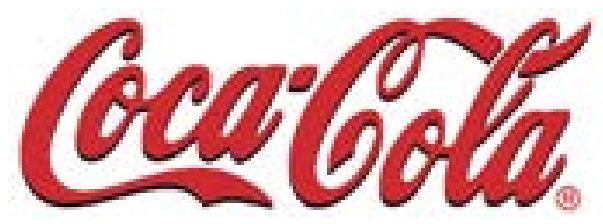

1.1.2 Icónico: Cuando se elabora un isotipo o icono que se convertirá en el distintivo figurativo de la empresa. Cuando una empresa pueda concentrar su identidad en una imagen simple puede responder cada vez más a la exigencia de los medios.
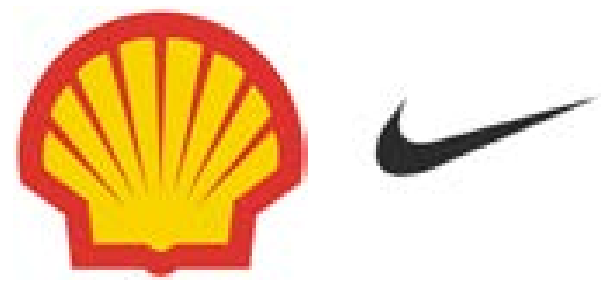

1.1.3 Cromático: Consiste en el color o los colores que la empresa adopta como distintivo emblemático. Estos formarán parte del logotipo comercial (la suma de la marca gráfica y el isotipo), además de convertirse en identidad de interiores y exteriores de locales, uniformes, decoraciones, etc.

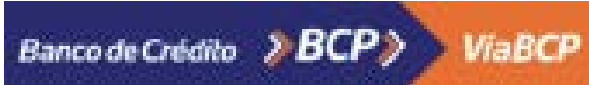

La identidad de una empresa se encuentra en los nombres, logotipos y colores que la empresa utiliza para distinguirse a si misma, a sus marcas y a sus empresa asociadas. En cierto modo éstos tienen el mismo propósito que los iconos religiosos, la heráldica, las banderas nacionales, $y$ otros simbolos: encierran en sí mismos sentimientos colectivos de pertenencia, y los hacen visibles. Además ofrecen hasta cierto punto, una garantía de calidad estándar consistente, y contribuyen a acrecentar la lealtad de los clientes y otros públicos objetivo. (2)

\subsection{Identidad no visual:}

Todo acto comunica y por lo tanto las acciones también se convierten en mensaje institucional. Podemos llamar identidad no visual al conjunto de comportamientos, prácticas, políticas, valores y tradiciones que posee una empresa de manera natural o artificial pero que la caracteriza e identifica, por lo tanto también la diferencia de las demás.

La identidad no visual está determinada por dos componentes que, controlados, puede transformar el funcionamiento, la efectividad y estado ánimo de los integrantes de una empresa:

\subsubsection{Cultura organizacional: Es un} sistema de valores compartidos y creencias que interactúan con la gente. La cultura cumple funciones importantes en la organización, incluyendo el diseño y el tipo de administración, transmite valores y filosofías, socializando a los miembros, facilita la cohesión del grupo y el compromiso de metas relevantes. 
Toda organización tiene una cultura espontánea formada. Es trabajo de la comunicación organizacional hacer que esta cultura no sea consecuencia del azar o las personalidades más fuertes. Para ello debemos planificar qué comportamiento queremos en la organización, las políticas, las tradiciones integradoras, etc. Si la cultura organizacional de una empresa es el fruto de la planificación podremos enrumbar las condiciones para lograr los objetivos últimos de la institución.

Una cultura organizacional debe contar con estrategias de comunicación orientadas a:

- Transmitir un sentimiento de identidad a los miembros de la organización.

- Facilitar el compromiso con algo mayor que el yo mismo.

- Reforzar la estabilidad social.

- Ofrecer premisas reconocidas y aceptadas para la toma de decisiones.

- Lograr un cambio más duradero en la organización.

- Crear la necesidad de mejorar la organización.

- Facilitar los cambios de métodos y estilos por medio del autoconocimiento.

- Crear la necesidad de modificaciones conductuales y adquisición de nuevas habilidades

- Lograr mayor control de los recursos y organización integral.

- Movilizar los recursos humanos en la identificación de problemas y búsqueda de soluciones creativas.

- Crear capacidad de autodiagnóstico y autodiseño de la estrategia empresarial.
La implicación del personal en el estudio de la cultura organizacional es un factor necesario, ya que está dirigido a la comprensión de los procesos que se dan en ellas para accionar hacia el mejoramiento de los resultados de las mismas. Para que una organización conozca sus potencialidades y pueda decidir qué alternativa estratégica tomar basada en un análisis realista, deberá estudiar y comprender su propia cultura.

\subsubsection{Clima organizacional}

"El comportamiento de un trabajador no es una resultante de los factores organizacionales existentes, sino que depende de laspercepciones que tenga el trabajador de estos factores.

Sin embargo, estas percepciones dependen en buena medida de las actividades, interacciones y otra serie de experiencias que cada miembro tenga con la empresa. De ahi que el Clima Organizacional refleje la interacción entre características personales y organizacionales.

Los factores y estructuras del sistema organizacional dan lugar a un determinado clima, en función a las percepciones de los miembros. Este clima resultante induce determinados comportamientos en los individuos. Estos comportamientos inciden en la organización, y por ende, en el clima." (5)

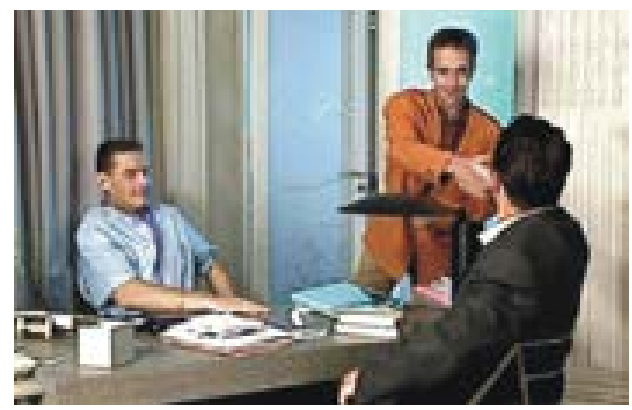


El clima es el conjunto de características del medio ambiente de trabajo. Estas son percibidas directa o indirectamente por los trabajadores que se desempeñan en ese medio ambiente y tiene repercusiones en el comportamiento laboral.

Desde una óptica científica, el clima es una variable interviniente que media entre los factores del sistema organizacional y el comportamiento individual. Estos factores son relativamente permanentes en el tiempo y se diferencian de una organización a otra y de una sección a otra dentro de una misma empresa.

Las percepciones y respuestas que abarcan el clima organizacional se originan en una gran variedad de factores, como liderazgo, prácticas de la Dirección, supervisión, etc.

El clima organizacional repercute sobre las motivaciones de los miembros de la organización y sobre su correspondiente comportamiento. Este comportamiento tiene obviamente una gran variedad de consecuencias para la organización como, por ejemplo, productivad, satisfacción, rotación, adaptación, etc.

Litwin y Stinger postulan la existencia de nueve dimensiones para medir el clima existente en una determinada empresa. La estrategia de comunicación corporativa deberá tomar en cuenta el clima sin el cual será imposible tener control sobre la cultura corporativa, la comunicación interna y la identidad.

- Estructura. La percepción que tienen los miembros de la organización acerca de las reglas, procedimientos, trámites y otras limitaciones en el desarrollo de su trabajo.

- Responsabilidad (empowermen ). Es el sentimiento de los miembros de la organización acerca de su autonomía en la toma de decisiones.

- Recompensa. Corresponde a la percepción de los miembros sobre la adecuación de la recompensa recibida por el trabajo bien hecho.

- Desafío. Corresponde al sentimiento que tienen los miembros de la organización acerca de los desafíos que impone el trabajo.

- Relaciones. Es la percepción por parte de los miembros de la empresa acerca de la existencia de un ambiente de trabajo grato y de buenas relaciones sociales.

- Cooperación. Es el sentimiento de los miembros de la empresa sobre la existencia de un espíritu de ayuda de parte de los directivos, y de otros empleados del grupo.

- Estándares. Es la percepción de los miembros acerca del énfasis que pone las organizaciones sobre las normas de rendimiento.

- Conflictos. Es el sentimiento del grado en que los miembros de la organización, tanto pares como superiores, aceptan las opiniones discrepantes y no temen enfrentar y solucionar los problemas. 
- Identidad. Es el sentimiento de pertenencia a la organización. Es un elemento importante y valioso dentro del grupo de trabajo.

Cuando los anteriores nueve aspectos se encuentran armonizados en un clima saludable, se obtendrá de los trabajadores la mejor disposición para el trabajo en equipo y con mejores actitudes dentro de la empresa.

\section{Imagen corporativa}

La imagen corporativa es el conjunto de percepciones que se tiene de la empresa. Mientras que la identidad es concreta, controlable y modificable, la imagen es subjetiva, difícil de controlar y también de modificar.

La imagen corporativa se proyecta principalmente a través de los medios masivos, aunque también se fortalece con acciones en la comunidad que vayan introduciendo valores en la opinión pública con respecto a la empresa.

Existen dos tipos de imagen corporativa. La promocional, que se desarrolla con la intensión de obtener una reacción inmediata del público, adquiriendo los productos o servicios que ofrece la institución; y la imagen motivacional, que se desarrolla con el objeto de orientar la opinión del público hacia metas de identificación o empatía entre la institución y el público target. (3)

\subsection{La imagen promocional}

Se desarrolla con estrategias de publicidad y marketing. Se piensa que en el mundo moderno, ningún producto puede sobrevivir sin imagen publicitaria.

Estaimagen está compuesta principalmente por las percepciones asociadas a la venta de un producto, la difusión de sus características, sus valores diferenciales, sus ventajas con respecto a la competencia, sus estrategias creativas para lograr "afinidad comercial" con el posible cliente, etc.

La posición... está determinada por los atributos o creencias del consumidor sobre la marca (esto es, su imagen del producto). Evidentemente, estas percepciones estarán influenciadas por las peculiaridades objetivas del producto, pero otros factores, principalmente la publicidad, desempeñarán un papel fundamental en la comunicación de la imagen de marca (4)

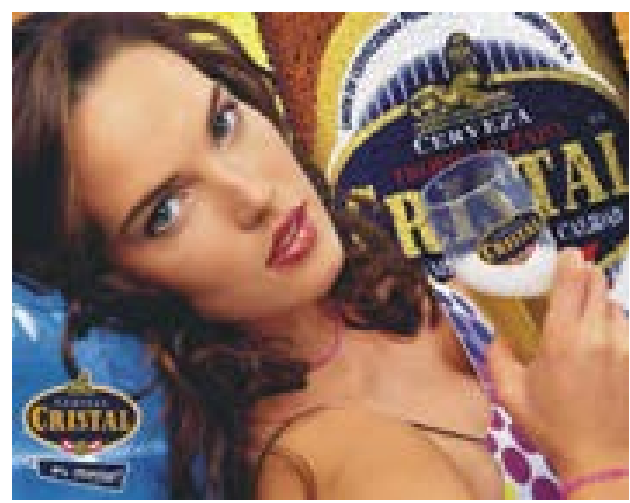

La asociación de un producto a los "deseos" del consumidor y la realización de recordatorios de marca, son el ejemplo típico de la imagen promocional

\subsection{La imagen motivacional}

Se proyecta con campañas de propaganda, campañas de relaciones públicas y relaciones con la comunidad. 
La imagen es lo que los marketeros llaman "posicionamiento", es decir, el lugar que ocupa una marca en la mente de las personas, los valores que surgen de manera espontánea cada vez que escucha mencionar el nombre de la empresa o ve su logotipo.

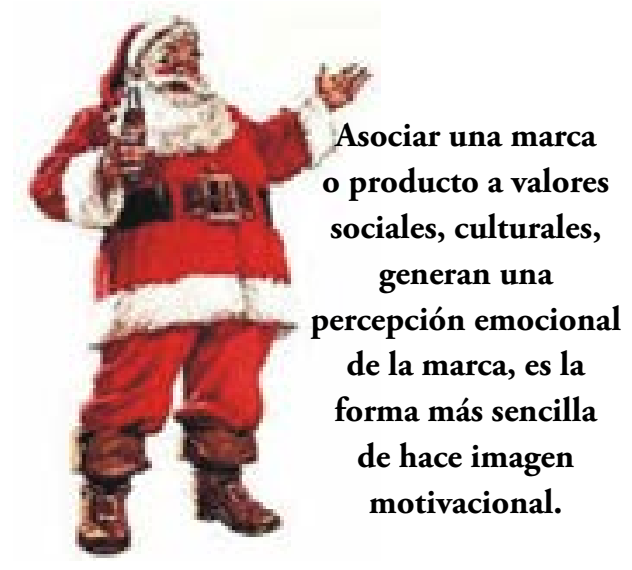

\section{Ejemplos de construcción de la identidad visual}

Teniendo en claro los conceptos antes mencionados de identidad e imagen corporativa, realizaremos un recorrido por algunos ejemplos de identidad visual basados en casos reales. Analizaremos sólo los criterios de comunicación sustentados en el diseño de tres isotipos realizados por Félix Beltrán* y cuatro logotipos comerciales.

Empresa: Películas de la Cruz Roja Cubana

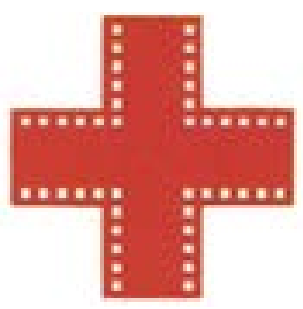

\section{Análisis visual:}

Este isotipo está caracterizado por la síntesis de sus elementos. Este logotipo pretendía servir de identidad visual a un festival de películas de la Cruz Roja Internacional. Beltrán mezcló ambos conceptos y decoró los bordes de la cruz con pequeños recuadros para asemejarse a una película de celuloide. Mencionamos entonces el primer requisito de un isotipo: síntesis.

\section{Empresa: Sandor, danzas de Nueva York}

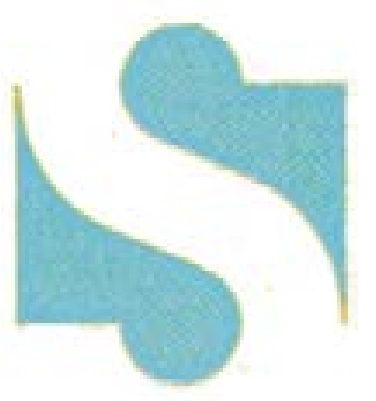

Análisis visual:

Este isotipo fue diseñado para un academia de danza en Nueva York y nos presenta una secuencia de formas con significado. La forma comunica por semejanza y sensación.

Las figuras coloreadas asemejan una pareja de baile, los vacíos forman la inicial de Sandor, haciendo énfasis además por sus formas curvas a la flexibilidad, un valor inherente a la danza. Nótese que la $S$ en realidad no existe a pesar de ser visible. Mencionamos el segundo requisito: Comunicación a través de las formas. 


\section{Análisis Visual:}

La principal característica de este logotipo comercial es la redundancia. Está repleto de asociaciones culturales al Perú o a lo peruano, repitiéndose y superponiéndose. La iconografía inca en el fondo azul, las murallas moche flanqueando los lados, el mapa del Perú escondido detrás del nombre y la propia marca gráfica (Inca Kola) hacen alusión a lo peruano. Esta ha sido la estrategia publicitaria de la empresa por décadas. Este mismo concepto está redundantemente sintetizado en el logotipo.

\section{Empresa: Línea Aérea Faucett}

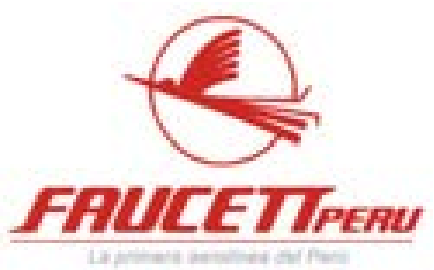

Análisis Visual:

La empresa Faucett (ya fenecida) utilizó un colibrí, como representación de sus aviones en ascenso, delante de un sol rojo como expresión de lo superior o ideal.

La tipografía fue diseñada para asemejarse a un avión que vuela de derecha a izquierda, visto de lado.

Los colores en este caso son el rojo y el blanco que asumen un significado patriótico nacional, siendo este el distintivo de su ofrecimiento comercial al público.

\section{Conclusión final}

Los logotipos comerciales son el centro de la identidad visual de las empresas, sintetizan sus valores y sus intenciones comerciales. Sin embargo, estos valores también están dados por la identidad no visual que fortalece la personalidad de una empresa, en el caso de la cultura corporativa y el clima organizacional.

La imagen corporativa es un esfuerzo de percepción por transmitir la identidad, los valores, las intenciones comerciales. Lo ideal es armonizar todo los elementos de imagen e identidad a fin de que se den al público los mensajes que se pretende dar.

\section{Nota}

* Félix Beltrán es un diseñador gráfico cubano graduado en la School of Visual Arts de Nueva York. Es famoso por sus exposiciones sobre arte visual y los cursos que dicta a nivel de toda latinoamérica

\section{Referencias bibliográficas}

(1) VAN RIEL, Cees. Comunicación Corporativa. Ed. Prentice Hall. Erasmus UNiversuty Rotterdam. España, 1998 (pág. 29)

(2) VAN RIEL, Cees. Comunicación Corporativa. Ed. Prentice Hall. Erasmus UNiversuty Rotterdam. España, 1998 (pág. 37)

(3) ESCOBAR, Jorge. La Comunicación Corporativa. Advenezuela. 
com, Escuela de Publicidad del Instituto Universitario de Nuevas Profesiones. Valencia, Venezuela, 2003 (pág. 7)

(4) SOLER, Pere. Estrategia de Comunicación en Publicidad y Relaciones Públicas. Ed. Gestión 2000. España, 1997 (pág. 36)

(5) MARTINEZ, Luis. Clima Organizacional. CETCOL, Colombia 2004 (pág. 3)

(6) RIES, Al. Las 22 Leyes Inmutables de la Marca. Ed. McGraw Hill. España, 2000 (pág. 146)

\section{BLIBLIOGRAFÍA}

ELÍAS, Joan; MASCARAY, José. Más allá de la comunicación interna. España, Ed. Gestión2000, 1998.

ESCOBAR FERNÁNDEZ, Jorge. La comunicación corporativa. Advenezuela.com, Escuela de Publicidad del Instituto Universitario de Nuevas Profesiones, Valencia, Venezuela, 2003
GARRIDO M., Francisco Javier. Comunicación estratégica. Barcelona, España, Ed. Gestión2000, 2004.

HOMS QUIROGA, Ricardo. La comunicación en la empresa. México, Ed. Iberoamericana, 1990.

LIBAERT, Thierry. El plan de comunicación organizacional. México, D.F., Ed. LIMUSA, 2005.

RIES, Al. Las 22 leyes inmutables de la marca. España, Ed. McGraw Hill, 2000.

SOLER, Pere. Estrategia de comunicación en publicidad y relaciones públicas. España, Ed. Gestión2000, 1997.

TAYLOR, Judith. La comunicación en el trabajo. España, Ed. GEDISA, 2002.

TOLELA, Michele. Administración mediante la comunicación: un enfoque organizacional. México, Ed. McGraw Hill, 1983.

VAN RIEL, Cees. Comunicación corporativa. España, Ed. Prentice Hall, Erasmus University Rotterdam, 1998. 